Website: http://revistas.lamolina.edu.pe/index.php/acu/index

(C) Universidad Nacional Agraria La Molina, Lima - Perú

\title{
Tipo de empaque y tiempo de almacenaje en frío en frutos de granadilla (Passiflora ligularis Juss)
}

\author{
Packing and cold storage time in sweet passion fruits (Passiflora ligularis Juss)
}

\author{
Viviana Noblecilla O; Guillermo Parodi M. ${ }^{1 *}$ \\ * Autor de correspondencia
}

\section{Resumen}

La presente investigación tiene como objetivo evaluar el efecto de distintos tipos de empaque en frutos de granadilla (Passiflora ligularis Juss) almacenados en frio a diferentes periodos de conservación. Para ello frutos de esta especie, previamente seleccionados en campo, se sometieron a tres condiciones de empaque; sin empaque (SE), empaque con film de PVC (EFP) y empaque en envases PET (EEP), conservándose a $10 \pm 1^{\circ} \mathrm{C}$ y H.R de $85-90 \%$ durante 15 y 30 días. Las evaluaciones realizadas en los frutos, concluido el almacenamiento en frío, como 5 días después de retirados del almacenaje en frío (simulación en anaquel a $24 \pm 2{ }^{\circ} \mathrm{C}$ y $80 \pm 5 \%$ de H.R.) fueron; Pérdida de peso (\%), Sólidos solubles totales (\%) (SST), Acidez titulable total (\%) (ATT), Azúcares reductores (mg de glucosa/mL de jugo) (AR) y Daño externo (grado 1; sin daño o menor al 0,1\% al grado 4; daño > al 5\%). El diseño estadístico fue DCA en factorial de 3 condiciones de empaque y 2 períodos de almacenaje. Los datos fueron sometidos a una prueba de Tukey $(p \leq 0,05)$ y el daño externo a la prueba estadística de Kruskal-Wallis $(\mathrm{p} \leq 0,05)$. Los resultados muestran que las granadillas tratadas con EFP y EEP se conservaron mejor hasta por 15 días. Observándose que los SST se redujeron a medida que el tiempo de conservación fue mayor sin ser afectados los ATT. Sin embargo el tratamiento EFP fue quien más estimuló la aparición de Colletotrichum sp., principal causante del daño externo en los frutos durante su almacenaje en frío, siendo mayor este daño 5 días después de retirados los frutos de frío y colocados a medioambiente. Daño que fue menor en los frutos tratados con EEP.

Palabras clave: Conservación; envoltura; empaques; passifloraceae; granada china; poscosecha.

\begin{abstract}
This research is aimed to study the effect of different packing and cold storage times on the postharvest behavior of sweet passion fruits. Fruits off the vine were tested in three packing conditions: Without packaging (SE), packaging with PVC film (EFP) and packaging PET (EEP). The fruits were stored at $10 \pm 1^{\circ} \mathrm{C}$ and R.H of $85-90 \%$ for 15 to 30 days. The evaluations were carried out at the end of cold storage and 5 days after the end of cold storage (shelf simulation at $24 \pm$ $2{ }^{\circ} \mathrm{C}$ and R.H. $80 \pm 5 \%$ ). It was analyzed; Weight loss (\%), Total soluble solids (\%) (TSS), Total treatable acidity (\%) (TTA), Reducing sugars (mg of glucose / mL of juice) (RA) and External damage (grade 1; unobservable or less than $0,1 \%$ to grade 4 , damage $>5 \%$ ). The statistical design was DCA in factorial of 3 packing and 2 storage periods. The data was analyzed by Tukey test $(\mathrm{p} \leq 0,05)$ and external damage by Kruskal-Wallis test $(\mathrm{p} \leq 0,05)$. The results showed that the treatments with EFP and EEP preserved the sweet passion fruits for 15 days. It was also found that the TSS decreased as the storage time increased, while the TTA was not modified. However, the fruits treated with EFP presented more external damage caused by the appearance of Colletotrichum sp. This damage was even greater 5 days after the fruits were removed from cold storage and placed in the environment. Damage that was minor in the fruits treated with EEP.
\end{abstract}

Keywords: Conservation; wrapped; packaging; passifloraceae; chinese grenade; postharvest.

\section{Introducción}

El país cuenta con 6,043 Ha en producción de granadilla (Passiflora ligularis Juss) siendo las regiones de Pasco, con 1,670 Ha y Junín, con 1,130 Ha, las que aportan el 66 $\%$ de la producción nacional de esta fruta con unas 37,600 toneladas (SIEA, 2017). Estas regiones actualmente proveen granadilla proveniente de plantaciones desarrolladas a partir de material vegetal de origen colombiano (Perez et al., 2006), aspecto que ha propiciado que diversos programas gubernamentales busquen colocar esta fruta en el mercado exterior (ANDINA, 2018). Actualmente, el uso de coberturas, películas o envases plásticos son una 
de las alternativas más usadas para asegurar un mayor tiempo de conservación poscosecha de diversos productos hortícolas (Yahia, 2009). Esta alternativa, se logra muchas veces gracias a la acción pasiva en la modificación de la atmósfera dentro del empaque por efecto de la actividad respiratoria del órgano vegetal (Ospina y Cartagena, 2008; Kader et al., 1989). En el caso de frutos de pasifloras esta técnica también ha sido ensayada, encontrándose que su uso, permitió mejorar la capacidad de conservación de estos frutos en poscosecha (Maniwara et al., 2015; Orjuela et al., 2011; Arjona et al., 1994). En granadilla, como lo es para otros frutos de pasifloras; la primera condición es contar con una temperatura adecuada de almacenaje, la cual debe ubicarse entre los 7 y $10{ }^{\circ} \mathrm{C}$ (Hernández y Fischer, 2009), en algunos casos se ha intentado conservarla a temperaturas de hasta $5{ }^{\circ} \mathrm{C}$ (Parodi, 1995; Villamizar et al., 1992), sin embargo, almacenajes mayores a los 28 días a esa temperatura; pueden llegar a ocasionarle daños por frío (Parodi, 1995). El uso de técnicas de almacenaje con atmósfera modificada en frutos de granadilla, ha logrado en algunos casos favorecer su conservación dependiendo esto del tipo de cobertura plástica utilizada (García, 2008; Arias et al., 2007). Uno de los principales beneficios del uso de coberturas plásticas en frutos de granadilla almacenados en frío, ha sido la reducción de su pérdida de peso. Encontrándose que, independientemente del tipo de cobertura; fueron la temperatura y/o el tiempo de almacenaje los factores que más influyeron en su conservación (Alvarado et al., 2011; Black y Ortega, 2005). Por otro lado, adicionalmente al uso de coberturas de polietileno de baja densidad (PEBD), también se sugiere como una alternativa para conservar los frutos de granadilla el utilizar envases de tereftalanato de polietileno (PET) (Parra et al., 2015). Estos envases tienen la particularidad de presentar un alto grado de cristalinidad, alta resistencia al desgaste y corrosión, gran resistencia química y térmica, una menor intensidad de transmisión de oxígeno y baja permeabilidad relativa en comparación con una película PEBD (Ospina y Cartagena, 2008). Por lo que resulta también de interés el evaluarlos como una alternativa para la conservación de frutos de granadilla.

El presente trabajo de investigación tuvo como objetivo estudiar el efecto de distintos tipos de empaques plásticos sobre el comportamiento poscosecha de frutos de granadilla en condiciones de almacenamiento en frío y diferentes períodos de conservación.

\section{Materiales y métodos}

\section{Lugar}

El ensayo se efectuó en el Laboratorio de Horticultura de la Facultad de Agronomía de la Universidad Nacional Agraria la Molina, distrito de La Molina-Lima.

\section{Materia prima}

Se tomaron frutos de campos comerciales de granadilla instalados con semilla de material vegetal obtenido de un ecotipo colombiano, cultivado por la Asociación de Productores de Granadilla Yanachaga-Chemillen en Yanajanca - San Daniel (Oxapampa). Los campos seleccionados estaban ubicados dentro de un ecosistema de Bosque húmedo-Premontano tropical a una altitud de 1,000 a 1,800 m.s.n.m con temperaturas, máximas promedio de $21,5^{\circ} \mathrm{C}$ y mínimas promedio de $13{ }^{\circ} \mathrm{C}$ y una precipitación anual de 1,600 - 1,700 mm. La cosecha se realizó a mediados del mes de junio del 2014. La recolección se hizo por la mañana entre las 8 y 11 am. Los frutos fueron recolectados con pedúnculo, el recorte se hizo utilizando una tijera de poda y siguiendo el procedimiento sugerido por Hernández y Fisher (2009). El grado de color y madurez de los frutos recolectados fue M4 y M5 (ICONTEC, 1997). Solo se consideraron frutos de categoría Extra y calibre A (diámetro y peso) según el Codex-Stan 316-2014 (FAO, 2014).

\section{Materiales y equipos}

Las coberturas plásticas fueron; Film autoadhesivo de PVC (Policloruro de Vinilo) espesor de 10 micras $\pm 3 \%$, permeabilidad al $\mathrm{O}_{2}\left(0,5\right.$ a $\left.10,9 \times 10^{16} \mathrm{~mol} \cdot \mathrm{mm}^{-2} \cdot \mathrm{s}^{-1} \mathrm{~Pa}^{-1}\right)$, permeabilidad al $\mathrm{CO}_{2}\left(1,6\right.$ a 5,1 x $\left.10^{16} \mathrm{~mol} \cdot \mathrm{mm}^{-2} \cdot \mathrm{s}^{-1} \mathrm{~Pa}^{-1}\right) \mathrm{y}$ permeabilidad al vapor de agua (2 a $30 \times 105$ mol.m $\left.\mathrm{m}^{-2} \mathrm{~s}^{-1}\right)$. Envases de material PET (Tereftalanato de polietileno), espesor $0,2-0,7 \mathrm{~mm}$ y dimensiones $13,5 \mathrm{~cm}$ x $9 \mathrm{~cm}$ x $19 \mathrm{~cm}$ con 6 agujeros de ventilación en la base de $0,6 \mathrm{~mm}$ de diámetro, tapa tipo bisagra con 6 rendijas de ventilación de 2 x $0,5 \mathrm{~cm}$ y material de alta transparencia, permeable al $\mathrm{O}_{2}\left(0,05\right.$ a $\left.0,1 \times 10^{16} \mathrm{~mol} \cdot \mathrm{mm}^{-2} \cdot \mathrm{s}^{-1} \cdot \mathrm{Pa}^{-1}\right)$, permeabilidad al $\mathrm{CO} 2\left(0,2\right.$ a $\left.0,3 \times 10^{16} \mathrm{~mol} \cdot \mathrm{mm}^{-2} \cdot \mathrm{s}^{-1} \cdot \mathrm{Pa}^{-1}\right)$ y permeabilidad al vapor de agua ( 1 a 1,5 x 10 $\left.0^{5} \mathrm{~mol} \cdot \mathrm{mm}^{-2} \cdot \mathrm{s}^{-1}\right)$.

Los frutos de granadilla cosechados, recibieron una limpieza sencilla en la zona del pedúnculo con el fin de retirar cualquier remanente del cáliz, luego se trasladaron ese mismo día hacia el laboratorio en envases de poliestireno expandido. Cada fruto estuvo cubierto por una redecilla de espuma sintética tipo 'Mallalón'. Al llegar al laboratorio se almacenaron temporalmente a $10^{\circ} \mathrm{C}$ por 24 horas con el fin de reducir su actividad respiratoria y su temperatura de campo. Posteriormente, se les removió la redecilla de 'Mallalón', descartándose aquellos que presentaron algún defecto. Los frutos seleccionados se sometieron a distintos tratamientos siendo estos, tres tipos de empaques; Sin Empaque (SE), Empaque con Film de PVC (EFP) y Empaque en Envases PET (EEP) evaluados durante 2 tiempos de conservación 15 y 30 días. Lo cual generó un total de 6 tratamientos. Todos los tratamientos se colocaron a una temperatura de $10 \pm 0,5{ }^{\circ} \mathrm{C}$ y $85-90 \%$ de humedad relativa, dentro de una cámara de marca TORREY ${ }^{\circledR}$ de 0,41 m3 de capacidad con control de temperatura inteligente de 1 a $24{ }^{\circ} \mathrm{C}$. Los frutos fueron evaluados después de cumplido sus respectivos periodos de almacenamiento en frío, y también a los 5 días después de haberse retirado de su almacenaje en frío. En este último caso lo frutos se mantuvieron dentro de sus empaques pero en condiciones de ambiente a $24 \pm 2{ }^{\circ} \mathrm{C}$ y $80 \pm 5 \%$ de H.R, ello con la 
finalidad de conocer su comportamiento post-almacenaje. Las evaluaciones consideradas fueron; Pérdida de peso: Determinada en valor porcentual de pérdida mediante una balanza marca Ohaus modelo PA 3102, serie Pionner 3100 de $0,01 \mathrm{~g}$ de precisión. Sólidos solubles totales: Determinado en grados brix con un refractómetro digital de mano marca Atago modelo Pal-1. Acidez titulable total: Determinada en porcentaje por titulación del ácido cítrico con $\mathrm{NaOH}$ 0,1N sobre una muestra de $5 \mathrm{cc}$ de jugo, según Bósquez (1992). Azúcares reductores: Expresado en concentración de $\mathrm{mg}$ de glucosa $/ \mathrm{mL}$ de jugo, utilizando el método del ácido 3,5 dinitrosalicílico de acuerdo con lo establecido por Miller (1959), en base a lectura a $550 \mathrm{~nm}$ en espectrofotómetro marca Labomed modelo SP 22. Esta evaluación se realizó solamente al finalizar los tiempos de almacenaje en frío y Daño externo: El cual se cuantificó utilizando la siguiente fórmula; en base a escala modificada de acuerdo a propuesta planteada por Zambrano (2013) indicada en la Tabla 1. Nivel de daño en la epidermis $=[\Sigma$ $\left(\mathrm{N}^{\circ}\right.$ frutos con igual grado de daño) $*($ Grado $\left.)\right] / \mathrm{N}^{\circ}$ total de frutos evaluados

Tabla 1. Escala de Grados y Severidad de Daño de Epidermis en Frutos de Granadilla (Passiflora ligularis Juss)

\begin{tabular}{ccc}
\hline Grado & $\begin{array}{c}\text { Severidad del } \\
\text { daño en la } \\
\text { epidermis del } \\
\text { fruto (\%) }\end{array}$ \\
\hline 1 & 0,0 a 0,1 & Daño no visible o menor al $0,1 \%$ \\
2 & $+0,1$ a 1,0 & Hay daño $>0,1 \%$ y no mayor al $1,0 \%$ \\
3 & $+1,0$ a 5,0 & Hay daño $>1,0 \%$ y no mayor al $5,0 \%$ \\
4 & $+5,0$ & Hay daño $>5,0 \%$ \\
\hline
\end{tabular}

\section{Análisis estadístico}

El diseño estadístico fue DCA dispuesto en arreglo factorial de 3 condiciones de empaque por 2 tiempos de conservación. Cada tratamiento estuvo conformado por 3 repeticiones con 3 observaciones (frutos) por repetición. Así mismo, se utilizó una prueba de comparación de medias de Tukey $(\mathrm{p} \leq 0,05)$ para las evaluaciones físico- químicas, así como una prueba no paramétrica de Kruskal-Wallis $(\mathrm{p} \leq 0,05)$ para la evaluación de daño externo. El análisis de datos se realizó utilizando el programa estadístico InfoStatStatistical desarrollado por Di Riezo et al. (2008).

\section{Resultados y discusión}

Porcentaje de Pérdida de peso

La pérdida de peso en los frutos de granadilla tanto a los 15 como a los 30 días se dio por efecto del tipo de empaque y el tiempo de almacenamiento, estas diferencias fueron apreciables también por efecto de la interacción tipo de empaque y tiempo de almacenaje siendo la pérdida de peso influenciada por el tiempo de conservación en relación con el tipo de empaque (Figura 1), de tal forma que los frutos de granadilla almacenados en frío y tratados con EFP presentaron menos pérdida de peso a los 15 días $(0,69 \%)$ y a los 30 días (1,54\%) siendo esta pérdida estadísticamente similar entre estos dos tiempos cuando se usó el empaque EFP, mientras que la pérdida de peso de los frutos tratados con SE si presentaron diferencias tanto a los 15 días $(0,86 \%$ de pérdida de peso) como a los 30 días de almacenamiento (2,12\% de pérdida de peso), comportamiento que también tuvieron los frutos de granadilla tratados con EEP almacenados por 15 días $(1,12 \%$ de pérdida de peso) al compararse con los almacenados por 30 días $(2,85 \%$ de pérdida de peso). La evaluación de los tratamientos 5 días después de retirados los frutos del almacenaje en frío (Figura 2), presentó diferencias estadísticas solo por efecto del tipo de empaque y donde el tratamiento con SE fue el que generó la mayor pérdida de peso $(2,16 \%)$, diferenciándose significativamente, esta pérdida de peso de la presentada por los frutos con empaque EFP (1,20\%) y empaque $\operatorname{EEP}(1,66 \%$ ) quienes tuvieron una menor y muy similar intensidad de pérdida de peso. El comportamiento de la pérdida de peso de los frutos de granadilla durante el almacenaje en frío en el presente ensayo, mostró la misma tendencia encontrada en otras investigaciones realizadas con frutas de esta especie que fueron tratadas con coberturas de polietileno y almacenadas en frío (Alvarado et al., 2011; Black y Ortega, 2005). Esta pérdida de peso se presenta de manera notoria a medida que el tiempo de almacenaje es mayor y depende en principio del tipo y características del empaque o cubierta plástica utilizada en estos frutos (Arias et al., 2007; Black y Ortega, 2005), aspecto que también fue observado en frutos de otras pasifloras que recibieron o no una cobertura plástica y fueron almacenadas en frío (Arjona et al., 1994). Es reconocido que el uso de envases, cubiertas o films plásticos utilizados como técnicas de empaque en productos vegetales generan, dependiendo del grado de permeabilidad al $\mathrm{O}_{2}$ y $\mathrm{CO}_{2}$; una modificación de la atmósfera dentro del empaque, que contribuye a una reducción sustancial de la tasa respiratorio, transpiratoria y de síntesis de etileno en los frutos, logrando que se reduzca la pérdida de peso durante el almacenaje (Yahia, 2009; Kader, et al., 1989). El hecho de observar una mayor pérdida de peso en los frutos tratados con SE y con EEP puede estar relacionada al hecho que los frutos tratados con SE y EEP tuvieron mejores condiciones de intercambio de gases con el ambiente de almacenaje y que en el caso de los frutos tratados con EEP ello estuvo relacionado con la cantidad y características de las perforaciones propias del empaque, comportamiento que Alvarado et al. (2011) observaron durante el almacenamiento de frutos de granadilla tratados con coberturas de polietileno de baja densidad a las cuales se le aplicaron perforaciones. Comportamientos similares han sido reportados en frutos de maracuyá tratadas con coberturas plásticas con perforaciones y almacenados en frio (Saenz et al., 1991). De hecho, la magnitud en la pérdida de peso observada en los frutos de maracuyá sin cobertura se relaciona principalmente con el incremento de la tasa respiratoria y la más rápida síntesis de etileno (Maniwara et al., 2015; Arjona et al., 1994). Es interesante, sin embargo, indicar que los valores de pérdida de peso registrados en el presente estudio fueron mucho menores 
que los reportados en otros trabajos de investigación en los que también se utilizaron coberturas plásticas en frutos de granadilla (Alvarado et al., 2011; Black y Ortega, 2005).

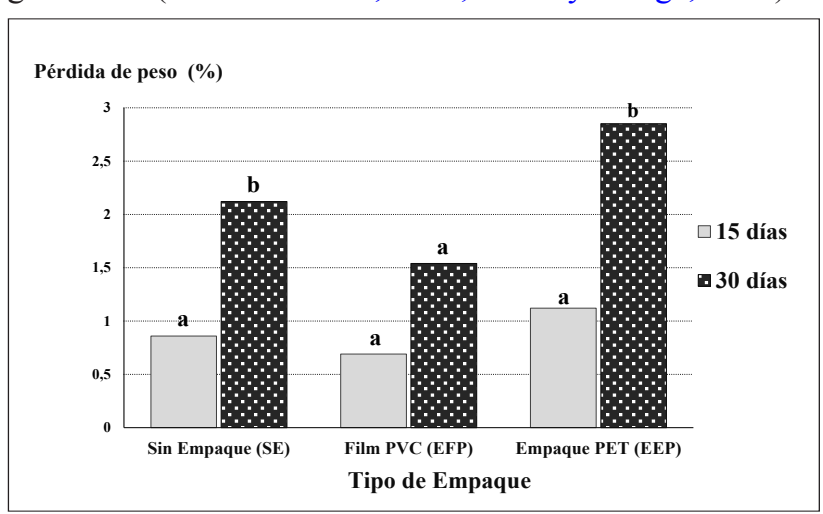

Figura 1. Pérdida de peso en frutos de granadilla (Passiflora ligularis Juss) por efecto del tipo de empaque en los distintos tiempos de almacenamiento en frío

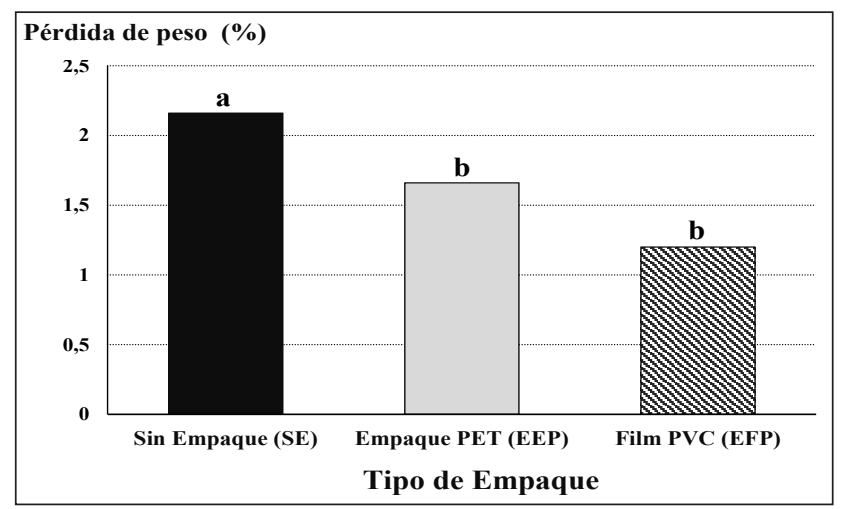

Figura 2. Pérdida de peso en frutos de granadilla (Passiflora ligularis Juss) por efecto del tipo de empaque a los 5 días después de retirados del almacenamiento en frío

\section{Sólidos solubles totales (SST)}

Los SST solo tuvieron diferencias estadísticas por efecto del tiempo siendo más significativo ello en los frutos de granadilla almacenados por 15 días (Tabla 2). Los SST en frutos de granadilla sometidos a almacenaje en frío no siempre han presentado una misma tendencia, en algunos casos los SST se han incrementado a medida que el periodo de conservación fue mayor (Cerdas y Castro, 2003), en otros casos no se modificaron a lo largo del tiempo de conservación (Parodi 1995; Villamizar et al.,1992) y en otros, tal como se observó en el presente ensayo, se reducen conforme el tiempo de almacenaje es mayor; siendo esta tendencia similar a la observada por otros investigadores (Alvarado et al., 2011; Mogollón et al., 2010) y parecido al mostrado por los frutos de otras pasifloras almacenadas en frío con o sin coberturas plásticas (Maniwara et al., 2015; Orjuela et al., 2015; Mohammed, 1993; Saenz et al., 1991). Esta disminución de los SST en los frutos de granadilla estaría relacionada con un aumento en su actividad respiratoria, generándose en este caso un consumo de sustrato respiratorio (carbohidratos) con la intención de sostener su energía vital durante el almacenaje (Alvarado et al., 2011). En algunos casos la disminución de estos compuestos no solo es producto de la respiración aeróbica sino incluso de procesos anaeróbicos por efecto del tipo de empaque (Maniwara et al., 2015). En el caso de los frutos de granadilla la predominancia de sacarosa y posteriormente su hidrólisis en fructosa y glucosa durante su maduración, resultan siendo los principales componentes de los SST (Espinosa et al., 2015). Frutos de maracuyá amarillo sometidos a almacenaje en frío redujeron sus contenidos de fructosa y glucosa entre un $15 \%$ hasta un $18 \%$, siendo esta reducción más apreciable desde los 15 hasta los 30 días de conservación y principalmente en aquellos que recibieron cobertura plástica (Arjona et al., 1994); En tal sentido la disminución del contenido de sólidos solubles en los frutos de granadilla, respondería a una degradación de los azúcares presentes en el fruto por efecto de una mayor actividad metabólica producto de la respiración. Parte de esta respuesta está relacionada con las características de permeabilidad a los gases del tipo de empaque (Maniwara et al., 2015; Kader et al., 1989), pero también a la presencia de daño en los frutos, lo cual se hizo evidente en algunos tratamientos a los 30 días de conservación, condición que puede contribuir a incrementar la tasa respiratoria y reducir el contenido de solidos solubles en los frutos (Pantastico, 1984). Al evaluar el contenido de SST 5 días después de retirados los frutos de sus periodos de almacenaje en frío (Tabla 3). No se halló, diferencias estadísticas en los valores observados pero so se observó una tendencia a la reducción de los SST en los frutos almacenados por 30 días en relación con los almacenados por 15 días. Orjuela et al. (2015) hallaron una más rápida perdida del contenido de sólidos solubles en frutos de gulupa expuestos a medio ambiente y tratados con empaque plástico XTend en comparación con los almacenados en frío. Indicando que este comportamiento estaría asociado a una mayor actividad respiratoria y transpiratoria estimulada por las condiciones térmicas del medio ambiente donde se colocaron los frutos para simular la etapa en anaquel. Este comportamiento a medioambiente es coincidente con lo manifestado por otros autores que consideran a los cambios en la tasa respiratoria de los frutos de granadilla el principal factor asociado a la pérdida del contenido de los SST en granadilla y otros frutos de passifloras durante su almacenaje prolongado en poscosecha (Maniwara et al., 2015; Arjona et al., 1994)

\section{Acidez titulable total (ATT)}

El comportamiento de la ATT evaluada en los frutos de granadilla al término del almacenaje frío, mostró valores estadísticamente similares entre los distintos factores y tratamientos estudiados (Tabla 2). La tendencia de la ATT fue coincidente con la mencionada por Villamizar et al. (1992); quienes no encontraron prácticamente cambios de ésta a lo largo del tiempo que duró el almacenaje en frío de la granadilla, respuesta que también fue observada por 
Mogollón et al. (2010). En otros casos, la acidez titulable puede llegar a incrementarse a medida que el tiempo de almacenamiento es mayor (García et al., 2008). La ATT en granadilla se sustenta principalmente por el contenido de ácido cítrico (Espinosa et al., 2015) por lo tanto, su persistencia, durante su almacenaje en poscosecha; contribuirá a retrasar el daño ocasionado por los procesos oxidativos propios del comportamiento de estas frutas después de recolectados (Espinosa et al., 2015; Pantastico, 1984), aspecto que en granadilla puede resultar beneficioso en términos de asegurar una mayor duración de los frutos durante su conservación. Por otro lado, la ATT 5 días después de retirados los frutos de su almacenaje en frío, mostró diferencias estadísticas solo por efecto del tipo de empaque (Tabla 3). Los frutos tratados con SE presentaron un mayor contenido de ATT diferenciándose significativamente de los valores encontrados en los frutos tratados con EFP y EEP los cuales tuvieron menores contenidos de ATT pero con valores muy similares entre sí. El comportamiento de la ATT se sustentaría en que los frutos de granadilla al ser expuestos a una temperatura ambiental menos fría que la utilizada durante su almacenaje, incrementan de manera sustancial su tasa respiratoria y transpiratoria (Yahia, 2009). Siendo esta pérdida más apreciable cuando en los frutos de granadilla u otras pasifloras no reciben una cobertura plástica (Black y Ortega, 2005; Mohammed, 1993; Saenz et al., 1991) Al incrementarse la transpiración en los frutos de granadilla se genera una mayor pérdida de agua lo cual ocasiona una mayor concentración de la acidez en el jugo de estos frutos (Alvarado et al., 2011; García et al., 2008). En la presente investigación ello habría determinado un mayor contenido de la ATT en los frutos tratados con SE, que fue apreciable 5 días después de ser retirados de su almacenaje en frío. A ello se debe adicionar que los frutos tratados con EFP y con EEP tuvieron un mayor daño externo causado por patógenos, lo cual también pudo contribuir a que se genere la caída de la ATT, pues los procesos oxidativos propios de la respiración, se estimulan por efecto del deterioro que ocasiona la presencia de patógenos en poscosecha contribuyendo a acelerar la perdida de ácidos orgánicos presentes en los frutos (Pantastico, 1984).

\section{Azúcares reductores totales (AR)}

Los AR en los frutos de granadilla presentaron diferencias estadísticas por efecto de los tiempos de conservación como por la interacción entre tratamientos, observándose que en la interacción los frutos almacenados por 15 días mostraron diferencias significativas, relacionándose esta respuesta con el tipo de cobertura utilizada. En tal sentido, los frutos tratados con EFP y almacenados por 15 días tuvieron el mayor contenido de AR $(19,578 \mathrm{mg}$ glucosa / $\mathrm{mL}$ jugo) en comparación con los frutos de granadilla almacenados por igual tiempo pero sometidos a los tratamientos SE y EEP los cuales presentaron entre un 16,7 a 17,7 \% menos AR que los tratados con EFP (Figura 3). Este comportamiento coincide con el observado en frutos de maracuyá tratados con cobertura plástica en poscosecha los cuales presentaron un descenso en los niveles de fructosa y glucosa después de 15 días de almacenaje en frío (Arjona et al., 1984). Por otra parte, el comportamiento de los AR en granadilla puede verse influenciado por el tipo de cobertura usada en algunos casos favoreciendo

Tabla 2. Porcentaje de sólidos solubles totales y acidez titulable total en frutos de Granadilla (Passiflora ligularis Juss) a la salida de sus periodos de almacenaje en frío

\begin{tabular}{|c|c|c|c|c|c|c|}
\hline \multirow{2}{*}{ Tipo de cobertura } & \multicolumn{3}{|c|}{ sólidos solubles totales (\%) } & \multicolumn{3}{|c|}{ acidez titulable total $(\%)$} \\
\hline & 15 días & 30 días & Promedio & 15 días & 30 días & Promedio \\
\hline Sin empaque (SE) & 12,6 & 12,0 & 12,3 a & 0,328 & 0,372 & $\mathbf{0 , 3 2 7}$ a \\
\hline Film PVC (EFP) & 13,3 & 11,5 & $12,4 \mathrm{a}$ & 0,287 & 0,342 & 0,314 a \\
\hline Empaque PET (EEP) & 13,8 & 12,5 & 13,1 a & 0,395 & 0,342 & 0,368 a \\
\hline Promedio & 13,2 a & $12.0 \mathrm{~b}$ & & 0,337 a & 0,352 a & \\
\hline
\end{tabular}

Tabla 3. Porcentaje de sólidos solubles totales y acidez titulable total en frutos de granadilla (Passiflora ligularis Juss) 5 días después de la salida de su almacenaje en frío

\begin{tabular}{cccc|ccc}
\hline \multirow{2}{*}{ Tipo de cobertura } & \multicolumn{2}{c|}{ sólidos solubles totales (\%) } & \multicolumn{3}{c}{ acidez titulable total (\%) } \\
\cline { 2 - 7 } & $\mathbf{1 5}$ días & $\mathbf{3 0}$ dias & Promedio & $\mathbf{1 5}$ días & $\mathbf{3 0}$ dias & Promedio \\
\hline Sin empaque (SE) & 12,6 & 11,9 & $\mathbf{1 2 , 2}$ a & 0,372 & 0,392 & $\mathbf{0 , 3 8 2} \mathbf{a}$ \\
Film PVC (EFP) & 12,7 & 11,5 & $\mathbf{1 2 , 1} \mathbf{a}$ & 0,278 & 0,309 & $\mathbf{0 , 2 9 3} \mathbf{b}$ \\
Empaque PET (EEP) & 12,9 & 11,9 & $\mathbf{1 2 , 9}$ a & 0,335 & 0,314 & $\mathbf{0 , 3 2 4} \mathbf{b}$ \\
\hline Promedio & $\mathbf{1 2 , 7}$ a & $\mathbf{1 1 . 8} \mathbf{a}$ & & $\mathbf{0 , 3 2 8} \mathbf{a}$ & $\mathbf{0 , 3 3 8}$ a & \\
\hline
\end{tabular}


una menor perdida de estos azúcares (Nayambati, 1984). De hecho el tipo de cobertura plástica, en este caso la EFP, que es la que presenta una menor permeabilidad a los gases del medio; estaría contribuyendo a reducir la tasa respiratoria de los frutos de granadilla durante los primeros 15 días de almacenaje en frío por un posible incremento en la concentración del $\mathrm{CO}_{2}$ y un cada vez menor presencia de $\mathrm{O}_{2}$ dentro del empaque (García, 2008), Lo cual determinaría un menor consumo de estos azúcares como sustrato respiratorio aspecto que es coincidente con el comportamiento de los azúcares reductores en frutos tratados con coberturas plásticas en poscosecha (Mahajan 2014; Kumar et al., 2012; Yahia, 1998; Kader et al., 1989). Esta respuesta no se observó a los 30 días de almacenamiento, dado que los tres tipos de cobertura presentaron concentraciones de AR muy similares estadísticamente. Sin embargo, es importante indicar que solo los frutos de granadilla tratados con EFP presentaron los valores más bajos de AR. En tal sentido es probable que dentro del empaque EFP se halla incrementando el $\mathrm{CO}_{2}$ y reducido cada vez más el $\mathrm{O}_{2}$, estimulándose el proceso de respiración anaeróbica (Maniwara et al., 2015), generándose un rápido descenso de los $\mathrm{AR}$ en éstos frutos por activación del ciclo de la glucolisis anaeróbica (Kader et al., 1989; Pantastico, 1984).

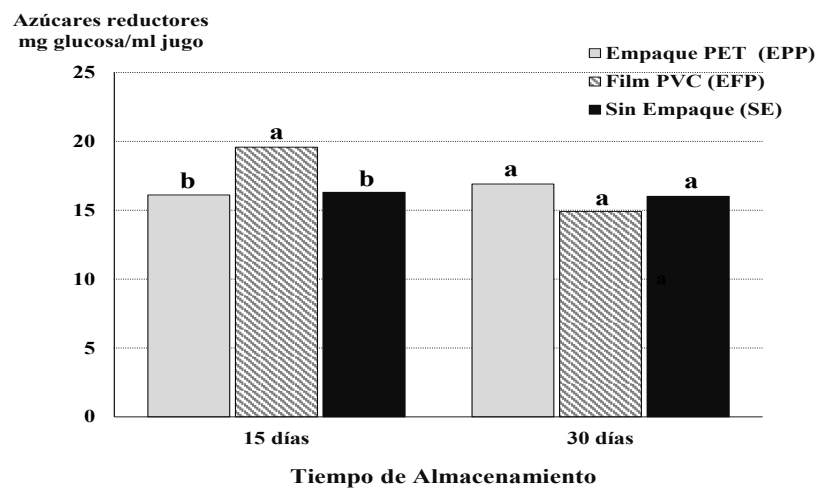

Figura 3. Azúcares reductores en frutos de granadilla (Passiflora ligularis Juss) por efecto del tipo de empaque en cada uno de los periodos de almacenamiento en frío

\section{Daño externo}

El análisis comparativo del daño externo en granadilla a la salida del almacenaje en frío presentó diferencias estadísticas entre tratamientos (Figura 4). El tratamiento SE con 15 días de almacenamiento, así como el tratamiento EEP evaluado a los 15 y 30 días de almacenamiento presentaron similar daño externo (grado 2 de daño), el cual no afectó más de $1 \%$ de la superficie del fruto. Este daño sin embargo fue notorio y muy similar en frutos tratados con SE almacenados por 30 días (grado 3,5 de daño) y en aquellos tratados con EFP por 15 y 30 días (grado 4 de daño) dicho daño afectó más del $4 \%$ de la superficie externa del fruto. La evaluación de los frutos, 5 días después de retirados de sus respectivos periodos de almacenaje en frío; presentó diferencias estadísticas entre tratamientos
(Figura 5). Los frutos con SE y EEP almacenados por 15 días, no registraron daño externo o este fue prácticamente imperceptible (grado 1 de daño), a diferencia de los frutos tratados con SE almacenados por 30 días (grado 3 de daño) así como los frutos tratados con EFP y con EEP almacenados en frío por 15 días y 30 días respectivamente, los cuales presentaron un daño muy similar y significativamente más alto (grado 3,5 de daño), en este caso los frutos de granadilla mostraron hasta cerca de un 5\% de deterioro en la epidermis. Sin embargo los frutos tratados con EFP y almacenados por 30 días, al evaluarse 5 días después de retirados de su conservación en frío; presentaron el mayor grado de daño externo (grado 4 de daño) observándose que el daño afecto más del $5 \%$ de la epidermis del fruto. El principal daño que se apreció en los frutos fue la presencia de manchas irregulares de color marrón oscuro sobre la epidermis. El análisis patológico indico que el daño fue ocasionado por el hongo Colletotrichum sp. (antracnosis), patógeno que contamina los frutos granadilla durante la fase de campo y que luego puede aparecer como un problema durante el almacenaje de ésta fruta en poscosecha (Hoyos y Castillo, 2015). Este problema se agudizó aún más en la granadilla tratada con EFP y EEP siendo notorio conforme el tiempo de almacenamiento fue mayor. El daño externo presentado en los frutos tratados con EFP almacenados por 15 como por 30 días y los tratados con EEP almacenados por 30 días, fue más elevado en parte debido a una mayor condensación de la humedad por incremento del vapor de agua dentro del empaque, aspecto que fue observado en el presente ensayo pero no medido. Este deterioro fue más evidente tras retirar los frutos de su almacenaje en frío y ser evaluados 5 días después. El uso de coberturas cerosas o plásticas en poscosecha puede generar en algunos casos, condiciones dentro del producto o empaque que contribuyen a un deterioro acelerado del órgano vegetal (Yahia, 2009; Kader et al., 1989), siendo una de ellas el incremento del vapor de agua dentro del empaque, propiciándose con esto la aparición de patógenos y/o desórdenes fisiológicos en las frutas u hortalizas tratadas (Arias et al., 2007; Kader et al., 1989). Esta condición ha sido observada en frutos de otras pasifloras tratadas con ceras o coberturas plásticas y almacenadas en frío (Mohamed, 1993; Arjona et al., 1992; Saenz et al., 1991; Nyambati, 1984), encontrándose en algunos casos que el tipo de cobertura usada en poscosecha estimuló la aparición de problemas fungosos en los frutos (Kumar et al., 2012; Mota et al., 2003). Es importante recalcar que la presencia de estos problemas fungosos no son consecuencia directa del empaque si no de una contaminación latente presente en el producto vegetal (Nyambati, 1984). En el caso de los frutos de granadilla y otros frutos de pasifloras la presencia de antracnosis es consecuencia de una contaminación que se inicia durante el manejo del cultivo en campo y que se manifiesta en poscosecha (Hoyos y Castillo, 2015). Siendo la prevención sanitaria en precosecha la forma más adecuada para controlar su aparición en poscosecha (García et al., 2007), no recomendándose en el manejo poscosecha de la granadilla, procedimientos de limpieza 
por lavado o desinfección en solución de los frutos a fin de evitar que éstos pierdan las ceras naturales propias de su epidermis (García, 2008).

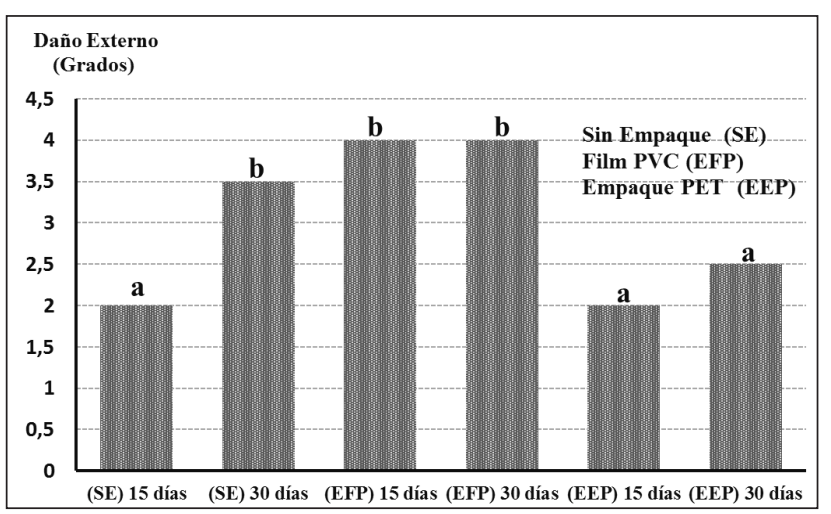

Figura 4. Daño externo en frutos de granadilla (Passiflora ligularis Juss) entre los distintos tratamiento al termino de los períodos de almacenamiento en frío

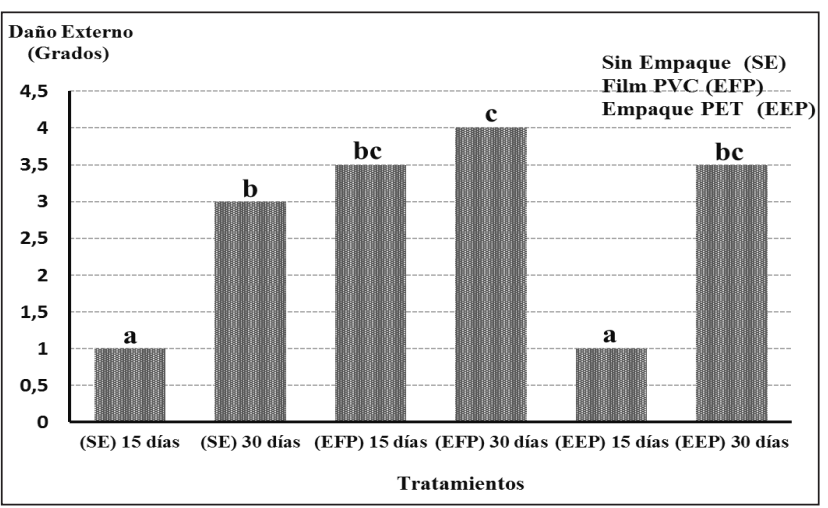

Figura 5. Daño externo en frutos de granadilla (Passiflora ligularis Juss) entre tratamientos 5 días después de la salida de los períodos de almacenamiento en frío

\section{Conclusiones}

El uso de EFP y EEP permitió una mejor conservación de los frutos de granadilla durante su almacenaje en frío por 15 días. A los 30 días solo los frutos tratados con EFP mostraron menor pérdida de peso. Durante el proceso de almacenaje se observó una reducción del contenido de SST conforme el tiempo de conservación fue mayor mientras que la ATT prácticamente no se modificó. Los AR se sostuvieron mejor en los frutos con EFP durante los primeros 15 días de conservación en frío. Sin embargo este tipo de envases estimuló una más rápida aparición de daño externo causado por Colletotrichum sp., siendo esto evidente desde los 15 días de conservación en frío, aspecto que se observó en los frutos tratados con EEP 5 días después de retirados de su de conservación en frío por 30 días.

\section{Literatura citada}

Alvarado, D; Marquez, L; Pretell, C.; Minchon, C. 2011. Influencia del empaque, temperatura y tiempo de almacenamiento sobre las características físicoquímicas y sensoriales de granadilla. Revista UCV-Scientia, 3(1): 101-109.

ANDINA. 2018. Perú inicia exportaciones de granadilla a Brasil. Artículo consultado el 12 de Febrero de 2018. Disponible en: http://www.andina.com.pe/agencia/ seccion-economia-2.aspx/img/noticia-peru-iniciaexportaciones-granadilla-a-brasil-697632.aspx

Arias, Y.A; Celis, H. V.; Varela, N.C. 2007. Determinación de las condiciones de un sistema de atmósferas modificadas para el almacenamiento y conservación de frutos de granadilla y uchuva. Tesis para Ingeniero Químico. Fundación Universidad de América. Facultad de Ingenierías. Departamento de Ingeniería Química. Bogotá. Colombia. 162 p.

Arjona, E.H; Matta F.B and James O. G. Jr. 1994. Wrapping in Polyvinyl Chloride Film Slows Quality Loss of Yellow Passion Fruit. Hortscience, 29(4):295-296.

Black, N.G y Ortega; L.F. 2005. Uso de atmósferas modificadas en la conservación de Babaco, Tomate de árbol y Granadilla. Informe del proyecto de investigación presentado para optar al título de Ingeniero Agropecuario. Escuela Politécnica del Ejército. Facultad de Ciencias Agropecuarias I.A.S.A "Grad. Carlomagno Andrade Paredes" Sangolquí. Ecuador. 163p.

Bósquez, E. 1992. Manual de prácticas de laboratorio de fisiología postcosecha de frutas y hortalizas. 1era ed Universidad Autónoma Metropolitana. Iztapalapa, México-D.F. MX. 74 p.

Cerdas, M. y Castro, JJ. 2003. Manual práctico para la producción, cosecha y manejo pos cosecha del cultivo de granadilla (Pasiflora ligularis Juss). San José, Costa Rica. FITTACORI. 12-50p.

Di Rienzo, J.A; Casanoves, F; Balzarini, M.G.; Gonzalez, L.; Tablada, M; Robledo, C.W. 2008 . InfoStat, versión 2008, Grupo InfoStat, FCA, Universidad Nacional de Córdoba. Argentina.

Espinosa, D.S; Pérez, W.H; Hernández, M.S; Melgarejo, L.M; Miranda, D; Fischer, G.; Fernández, J.P. 2015 Capítulo 3. Caracterización fisicoquímica, fisiológica y bioquímica del fruto de granadilla (Passiflora ligularis Juss). In Granadilla (Passiflora ligularis Juss) caracterización ecofisiológica del cultivo. Ed. L M Melgarejo. Universidad Nacional de Colombia (Sede Bogotá). Facultad de Ciencias: Colciencias: CEPASS-. Bogotá. CO. 91-118p.

FAO (Organización de las Naciones Unidas para la Alimentación y la Agricultura) 2014. Norma para las Frutas de la Pasión CODEX STAN 316-2014. 5p. 
García, M. C. 2008. Manual de manejo cosecha y poscosecha de granadilla. Bogotá. Corpoica, CO. 100 p.

García, H.R; Brito, B; García, M.C. 2008. Desarrollo tecnológico para el fortalecimiento del manejo postcosecha de frutales exóticos exportables de interés para los países andinos: uchuva (Physalis peruviana L.), granadilla (Passiflora ligularis L.), y tomate de árbol (Cyphomandra betacea (Cav) Sendt). Informe FONTAGRO. Proyecto FTG 14-03. Convenio RF0306-RG. Informe Final (Octubre 2008). CORPOICA (Corporación Colombiana de Investigación Agropecuaria)/ I N I A P (Instituto Nacional Autónomo de Investigaciones Agropecuarias)/CIAT (Centro Internacional De Agricultura Tropical)/ PROEXANT (Corporación Promoción de Exportaciones Agrícolas No Tradicionales)/ CIRAD (Centro Internacional de Investigación Agro-alimentario para el Desarrollo). $137 \mathrm{p}$.

García Lozano, J; Chamorro, L.E; Floriano, J.A; Vera, L.F; Segura, J.D. 2007. Enfermedades y plagas del cultivo de la granadilla (Passiflora ligularis) en el departamento del Huila. Corpoica - Huilaunido. Boletin técnico. C.I Nataima. 24 p.

Hernández, M.S. y Fischer, G. 2009. Cosecha y poscosecha en las frutas pasifloráceas. In Cultivo, poscosecha y comercialización de las pasifloráceas en Colombia: maracuyá, granadilla, gulupa. 1era ed. D, Miranda; Fischer, G; Carranza, C; Magnitskiy, S; CasierraPosada, F; Piedrahíta, W. y Flórez, L.E. (Eds). Sociedad Colombiana de Ciencias Hortícolas. Ruben's Impresores Editores. Bogotá. CO. 267-281p.

Hoyos, L. y Castillo, S. 2015. Capítulo 5. Enfermedades en granadilla (Passiflora ligularis Juss). In Granadilla (Passiflora ligularis Juss): Caracterización ecofisiológica del cultivo. Ed. L M Melgarejo. Universidad Nacional de Colombia (Sede Bogotá). Facultad de Ciencias: Colciencias: CEPASS-. Bogotá. CO. 153-176p.

ICONTEC (Instituto Colombiano de Normas Técnicas y Certificación) 1997. NTC 4101. Frutas Frescas. Granadilla. Especificaciones. Bogotá. 14 p.

Kader, A.A.; Zagory, D.; Kerbel, E; Wang, C.Y. 1989. Modified atmosphere packaging of fruits and vegetables. Jounal Critical Reviews in Food Science and Nutrition, 28 (1):1-30.

Kumar, R.; Misra, K.K; Mishra, D.S; Brijwal, M. 2012. Packaging Material and Storage Life of Fruits. Environment \& Ecology, 30 (3C): 1177-1184.

Mahajan, B.V.C; Dhillon,W; Mahesh, K; Bikramjit S. 2015. Effect of different packaging films on shelf life and quality of peach under super and ordinary market conditions J.Food Sci. Technol., 52(6):3756-3762,

Miller, G. 1959. Use of dinitrosalicylic acid reagent for determination of reducing sugar. Anal.Chem. 31: 426428.
Mogollón, M; Parra, A; Hernández, J; Acuña, J. 2010. Estudio fisiológico de la poscosecha de granadilla (Passiflora ligularis Juss) bajo dos condiciones de almacenamiento. In Primer Congreso Latinoamericano de Pasifloras (2010, Neiva, Huaila, Colombia). Memoria 1era ed. Huila. CO. 108p.

Mohammed, Majeed. 1993. Storage of Passion Fruits in Polimeric Films. Proc. Interamer. Soc. Trop. Hort., 37: 85-88,

Mota, W.F da; Chamhum, L.C; Cecon, P.R.; Finger, F.L. 2003. Waxes and plastic film in relation to the shelf life of yellow passion fruit. Scientia Agricola, 60(1): 51-57.

Nyambati, G.M. 1984. Use of semi-permeable membranes to increase the shelf-life of passion fruits. Master's Thesis. Loughborough University Institutional Repository. London. United Kingdom. 107 p.

Orjuela, J.A; Pinilla, A.L; Rincón, J.R. 2002. Aplicación de la tecnología de atmósfera controlada para la conservación de la granadilla. Ingenieria, 7(2): 45-53.

Ospina, S. y Cartagena, J. 2008. "La atmósfera modificada: una alternativa para la conservación de los alimentos". Revista Lasallista de Investigación, 5 (2): 112-123.

Pantástico, ER. B. 1984. Fisiología de la post-recolección, manejo y utilización de frutas y hortalizas tropicales y subtropicales. 2da ed. México. CECSA. 663 p.

Parodi, G. 1995. Estudio del comportamiento de la granadilla (Passiflora ligularis Juss.) sometida a una baja temperatura de almacenamiento por diferentes períodos. Proc. Interamer Soc. Trop. Hort., 39:151155.

Parra, M; Rodríguez, A; Piedrahita C.A; Gordillo, A. 2015. Capítulo 8. Comercialización de granadilla (Passiflora ligularis Juss). In Granadilla (Passiflora ligularis Juss) caracterización ecofisiológica del cultivo. Ed. L M Melgarejo. Universidad Nacional de Colombia (Sede Bogotá). Facultad de Ciencias: Colciencias: CEPASS-. Bogotá. CO. 231-248p.

Pérez, V; Albengrin, E; Roncal, Z; Berrospi, L; Quispe, E; Córdova, J; Rada, O. 2006. Estudio de mercado de los frutos de Granadilla, Palta, Lúcuma y Chirimoya. Fundación Peruana para la Conservación de la Naturaleza - Programa Selva Central- The Nature Conservancy TNC. Proyecto Parques en Peligro (PIP)/ USAID. Oxapampa-Perú. 29 p.

Saenz , M.V; Castro-Barquero, L; Gonzalez-Calvo, J. 1991. Efecto del empaque y la temperatura de almacenamiento sobre la vida poscosecha y la calidad de los frutos de maracuyá amarillo (Passiflora edulis var.flavicarpa). Agronomía Costarricense, 15(1/2): 7983.

SIEA (Sistema Integrado de Estadística Agraria). 2017. Documento consultado en Abril del 2018. Disponible en: http://siea.minagri.gob.pe/siea/?q=anuarioestadistico-de-producción-agricola-2017. 
Villamizar de Borrero, F; Gutiérrez, C; Pulido, A. 1992. La granadilla, su caracterización física y comportamiento postcosecha. Ingeniería e Investigación, (28), 14-23.

Yahia, E.M. 2009. Introduction. In Modified and controlled atmospheres for the storage, transportation, and packaging of horticultural commodities. Elhadi Yahia (eds). CRC Press Taylor \& Francis Group 6000 Broken Sound Parkway NW, Suite 300 Boca Ratón, FL. 1-16p.

Zambrano, L. 2013. Evaluación del efecto de coberturas naturales en el almacenamiento en frio de frutos de palto (Persea americana Mill) cultivar Hass. Tesis Ing. Agr. UNALM Lima. Perú. 53 p. 